\title{
Clinical implications of DMSA Scan in Childhood Acute Pyelonephritis
}

\section{Sun-Mi Huh, M.D. ${ }^{1}$ \\ Bo-Kyoung Park, M.D. ${ }^{1}$ Hyun-Mi Kang, M.D. ${ }^{1,2}$ Jung-Woo Rhim, M.D. ${ }^{1,2}$ Jin-Soon Suh, M.D. ${ }^{1}$ Kyung-Yil Lee, M.D. ${ }^{1,2}$}

Departments of Pediatrics', College of Medicine, The Catholic University of Korea, Seoul, Korea, Departments of Pediatrics', Daejeon St. Mary's Hospital, College of Medicine, The Catholic University of Korea, Daejeon, Korea

Corresponding author: Kyung-Yil Lee, M.D. Department of Pediatrics, Daejeon St. Mary's Hospital, College of Medicine, The Catholic University of Korea, 64 Daeheung-ro, Jung-gu, Daejeon 34943, Republic of Korea

Tel: $+82-42-220-9540$

Fax: +82-42-221-2925

E-mail: leekyungyil@catholic.ac.kr

Received: 27 September 2017

Revised: 30 September 2017

Accepted: 2 October 2017
This is an open-access article distributed under the terms of the Creative Commons Attribution Non-Commercial License (http:// creativecommons.org/licenses/by-nc/4.0/) which permits unrestricted non-commercial use, distribution, and reproduction in any medium, provided the original work is properly cited.

Copyright (C) 2017The Korean Society of Pediatric Nephrology
Purpose: This study aimed to evaluate the relationships between 99mTecniciumdimercaptosuccinic acid (DMSA) scan findings and clinical parameters including age and fever duration.

Methods: The positive rates for abnormal DMSA scans were analyzed according to the age of patients, fever duration prior to admission, and total fever duration. DMSA scan findings were divided into 3 categories: single defect, multifocal defects, and discrepant defects. We evaluated the detection rates of vesicoureteral reflux according to DMSA scan lesions.

Results: Among a total 320 cases, 141 (44.1\%) had abnormal DMSA scans. The infant group (0-1 year of age) had a shorter total fever duration, and a lower Creactive protein (CRP) value and DMSA positive rate $(39.8 \%$ vs. $60.6 \%, P=0.002)$ compared to children group (2-15 years of age). Patients with abnormal scans had a longer total fever duration and higher CRP compared to those with normal scans. The positivity rate of abnormal scans did not differ between the patients with a short fever duration prior to admission of $\leq 2$ days and those with longer fever duration of $\geq 3$ days. However, patients with longer total fever duration had a higher rate of abnormal DMSA scans $(P=0.02)$. Among cases with a single defect, multifocal defects, and discrepant defects, vesicoureteral reflux was observed in $22.4 \%, 60 \%$ and $70.6 \%$ of cases, respectively ( $P=0.004$ ).

Conclusion: Although DMSA scan has limitations in early diagnosis, DMSA scan findings may aid in the prediction of the severity of systemic inflammation and detection of vesicoureteral reflux.

Key words: Acute pyelonephritis, Urinary tract infection, Technetium 99m- dimercaptosuccinic acid, Vesicoureteral reflux, Child

\section{Introduction}

Acute febrile urinary tract infection (UTI) or acute pyelonephritis (APN) may be the most common systemic bacterial infection in childhood, especially in infants ${ }^{1)}$. Childhood APN has unique demographic characteristics; it mainly affects infants ( $0-1$ year of age) with a male predominance; and after 2 years of age, female predominance has been noted ${ }^{2}$. It is suggested that the immature immune status of infancy is related to the pathogenesis of $\mathrm{APN}^{3)}$. Although the pathogenesis of renal cell injury in APN remains unknown, it is believed that APN has a focus in the renal parenchyma, where uropathogens replicate extracellularly and produce toxic substances against the renal 
cells. Therefore, imaging studies such as 99mTechnetiumdimercaptosuccinic acid (DMSA) scan, renal ultrasonography, and occasionally computed tomography (CT) have been used to detect the focus of $\mathrm{APN}^{4)}$.

Many studies have reported that the DMSA scan aids in the detection of renal parenchymal lesions. However, the detection rates during the acute stage of the first febrile APN vary among study groups, and they are lower in infants compared to older children ${ }^{5}$. Moreover, it is known that abnormal findings on DMSA scans are found more often in patients with vesicoureteral reflux (VUR) than in those without VUR, and the positive rate correlates with the severity of $\mathrm{VUR}^{6}$. These findings suggest that the immune status of the host, size of the focus, i.e., severity of inflammation, and anatomical defects in kidneys may be associated with positive findings for abnormal defects in DMSA scans ${ }^{3)}$.

The main risk factor for recurrent APN is believed to be VUR, and early detection of VUR through voiding cystourethrogaphy (VCUG) may be helpful to prevent recurrent renal cell injury and subsequent chronic kidney disease ${ }^{7}$. However, it is not recommended to perform such invasive study in all patients diagnosed with first APN, and the role of a DMSA scan as substitute method for VCUG or in identification of patients requiring VCUG is also controversial $8,9)$.

The aim of this study was to evaluate the relationships between abnormal findings on DMSA scan and clinical parameters, in order to aid in the interpretation of the lesions observed on the scan, and to understand its role in detecting VUR, and showing the extent of inflammation in childhood APN.

\section{Materials and methods}

This was a retrospective study in children aged 15 years and under, who were admitted for febrile APN to The Catholic University of Korea Deajeon St. Mary's Hospital between January 2005 and December 2015. In this study, APN was diagnosed in patients who satisfied all of the following criteria: pyuria, positive urine culture results with a single pathogen growth of $>10^{5}$ colony forming units $/ \mathrm{mL}$, and C-reactive-protein (CRP) values of $>2.0 \mathrm{mg} / \mathrm{dL}$ at pre- sentation. Urine samples for examination were obtained via clean-catch method (sterile bag collected urine samples, mid-stream urine samples, or catheterized urine samples). Pyuria was defined as microscopic urinalysis showing $>10$ while blood cells (WBCs)/high power field, with/without positive leukocyte esterase, positive nitrites, or bacteriuria detected via an automatic urine analyzer. With respect to the other laboratory indices, WBC with differential, hemoglobin, platelet, erythrocyte sedimentation rates (ESR), total protein, albumin, and CRP at presentation were analyzed. Fever was defined as a body temperature of $\geq 38^{\circ} \mathrm{C}$ (measured based on tympanic temperature), and the first day of fever onset was regarded as the first day of illness.

All subjects underwent DMSA scan and renal ultrasonography (US) during hospitalization. A DMSA scan was performed according to the standard protocol at mean $2.8 \pm 1.2$ days after admission (range 2-6 days). Four static images obtained in the anterior, posterior, right posterior oblique, and left posterior oblique projections were used for analysis. A positive DMSA scan was defined as the presence of decreased radioactive cortical uptake in one or both kidneys. In addition, $>10 \%$ difference in the cortical uptake density between the two kidneys and/or a different size or shape of both kidneys were defined as discrepant lesions. All subjects were divided into 3 groups depending on their DMSA scan findings: a single defect, multiple defects (more than 1 defect) in one or both kidneys, and discrepant defects. On renal US, abnormal findings included dilatation of the collecting system (pelvic diameter $\geq 4 \mathrm{~mm}$ ) and hydronephrosis, difference in renal size of $>10 \%$, ureteral dilatation, or an increase in echogenicity. The severity of hydronephrosis was defined according to the Society for Fetal Urology classification. Voiding cystourethrography (VCUG) was not routinely performed. Indications for performing VCUG during the study period were as follows: severe abnormal US findings (>grade II), abnormal DMSA imaging and US findings, or multiple defects and/or discrepant defects in the DMSA scan. None of the male patients were circumcised, and there were 25 patients with recurrent UTI with/without urologic malformations, and only the data obtained from the first episode were included in this study. This study was approved by the institutional review board of Daejeon St. Mary's Hospital, College of Medicine, The Catholic University of Korea. 
www.chikd.org

\section{Statistical analysis}

Data are presented as the mean \pm standard deviation for normally distributed values. Categorical variables are expressed as frequencies and proportions. Differences between the groups were evaluated by Student's t test, and ANOVA test. Comparisons of categorical variables between the groups were evaluated by the $\chi^{2}$ test. Statistical analyses were performed using IBM SPSS version 20.0 (IBM Corp., Armonk, NY, USA). Statistical significance was defined as $P<0.05$.

\section{Results}

Among a total of 593 children admitted for pyuria and fever at The Catholic University of Korea Deajeon St. Mary's Hospital between January 2005 and December 2015, 320 patients with APN met the inclusion criteria.

\section{Comparison of clinical and laboratory indices between the infant and children groups}

Of the total 320 subjects, 230 patients were less than 1 year old (162 male and 68 female patients), 24 patients were 1 year old (11 male and 13 female patients), and 66 patients were aged between ages 2-15 years. The infant group (0-1 year old) had a distinct predilection for APN (79.4\%, 254/ $320)$ with male predominance $(2.1: 1,173 / 81)$. The number of patients in the children group (2-15 years old) was relatively even throughout each age, with female predominance $(1: 5,11 / 55)$.

The clinical and laboratory indices were compared between the infant group and the children group. The fever duration prior to admission (2.6 vs. 3.2 days, $P=0.03$ ) and total fever duration ( 3.6 vs. 5.2 days, $P<0.001$ ) were significantly greater in the children group, suggesting that the children group had a more severe degree of inflammation caused by APN compared to the infant group. There were no differences in urinalysis, including nitrite positivity in both groups. CRP value $(6.3 \mathrm{mg} / \mathrm{dL}$ vs. $8.5 \mathrm{mg} / \mathrm{dL}, P=0.01)$ was higher in the children group, but ESR was not different in both groups. Other laboratory indices such as WBC differentials (neutrophil, lymphocyte, and monocyte), hemoglobin, platelet, total protein, and albumin were different between the groups, but some of these parameters might be in part affected by the patients' age. The infant group showed a lower rate of DMSA positivity (39.8\%) compared to the children group $(60.6 \%, P=0.002)$ (Table 1).

\section{Comparison of clinical and laboratory indices} between the DMSA positive and negative groups

Among the total 320 patients, 141 patients had a positive DMSA scan (44.1\%). We compared the clinical and laboratory indices of the subjects according to DMSA positivity. There were no differences in male-to-female ratio, fever duration prior to admission (2.9 days vs. 2.7 days), the duration from the day of admission to the date of examination of a DMSA scan (2.7 days vs. 2.9 days), and urinalysis findings. However, the DMSA positive group was older, and had a longer total fever duration (4.5 days vs. 3.5 days, $P<$ $0.001)$ and a higher WBC $\left(18900 / \mathrm{mm}^{3}\right.$ vs $14,800 / \mathrm{mm}^{3}, P<$ $0.001)$ with higher neutrophil differential, CRP $(8.0 \mathrm{mg} / \mathrm{dL}$ vs. $5.8 \mathrm{mg} / \mathrm{dL}, P=0.001)$, and total protein $(6.6 \mathrm{~g} / \mathrm{dL}$ vs. 6.3 $\mathrm{g} / \mathrm{dL}, P=0.002)$ values compared to the DMSA negative group. These findings suggest that the DMSA positive group

Table 1. Clinical Comparison between the Patients with 0-1 and 2-15 Years of Age

\begin{tabular}{|c|c|c|c|}
\hline Age groups & $\begin{array}{l}0-1 \text { years } \\
(n=254)\end{array}$ & $\begin{array}{c}2-15 \text { years } \\
(n=66)\end{array}$ & $P$ value \\
\hline Male:Female & $173: 81$ & $11: 55$ & $<0.001$ \\
\hline \multicolumn{4}{|l|}{ Fever duration (days) } \\
\hline Prior to admission & $2.6 \pm 1.7$ & $3.2 \pm 2.3$ & 0.03 \\
\hline Total & $3.6 \pm 2.0$ & $5.2 \pm 3.1$ & $<0.001$ \\
\hline \multicolumn{4}{|l|}{ Laboratory finding } \\
\hline \multicolumn{4}{|l|}{ Urine } \\
\hline Leukocyte esterase, n (\%) & $240(94.5)$ & $60(90.9)$ & 0.247 \\
\hline Nitrite, n (\%) & $99(39)$ & $22(33.3)$ & 0.390 \\
\hline Bacteriuria & $218(85.8)$ & $55(83.3)$ & 0.542 \\
\hline \multicolumn{4}{|l|}{ Blood } \\
\hline $\mathrm{WBC}\left(/ \mathrm{mm}^{3}, \times 10^{3}\right)$ & $16.9 \pm 6.8$ & $15.4 \pm 5.4$ & 0.097 \\
\hline Neutrophil (\%) & $50 \pm 14$ & $73 \pm 12$ & $<0.001$ \\
\hline Lymphocyte (\%) & $37 \pm 14$ & $18 \pm 11$ & $<0.001$ \\
\hline Monocyte (\%) & $11 \pm 6$ & $8 \pm 3$ & $<0.01$ \\
\hline Hemoglobin (g/dL) & $10.9 \pm 1.1$ & $12.2 \pm 1.2$ & $<0.001$ \\
\hline Platelet $\left(/ \mathrm{mm}^{3}, \times 10^{3}\right)$ & $375 \pm 102$ & $281 \pm 93$ & $<0.001$ \\
\hline $\operatorname{ESR}(\mathrm{mm} / \mathrm{h})$ & $55 \pm 32$ & $61 \pm 32$ & 0.095 \\
\hline $\mathrm{CRP}(\mathrm{mg} / \mathrm{dL})$ & $6.3 \pm 4.4$ & $8.5 \pm 6.8$ & $<0.01$ \\
\hline Total protein (g/dL) & $6.2 \pm 0.5$ & $7.2 \pm 0.7$ & $<0.001$ \\
\hline Albumin (g/dL) & $4.1 \pm 0.3$ & $4.3 \pm 0.5$ & 0.027 \\
\hline DMSA positive, n (\%) & $101(39.8)$ & $40(60.6)$ & 0.002 \\
\hline
\end{tabular}

Abbreviations: WBC, while blood cell; ESR, erythrocyte sedimentation rate; CRP, C-reactive protein. 
had a more severe degree of inflammation caused by APN (Table 2).

\section{DMSA scan positive rates according to fever duration}

The patient group with abnormal defects on DMSA scans had a longer total fever duration and a higher laboratory indices including CRP value; therefore, we analyzed the positivity of a DMSA scan according to fever duration. There were no differences in the percentage of patients with a positive DMSA scan between patients who had a fever duration of $\leq 2$ days prior to admission $(40.9 \%, 72 / 176)$ and those with a longer fever duration ( $\geq 3$ days, 47.9\%, 69/144) $(P=0.15)$. With respect to the total fever duration, subjects were classified into 3 groups: patients with a fever duration of $\leq 4$ days, those with a fever duration of 5-7 days, and those with a fever duration of $\geq 8$ days. There was a statistical significance for a higher DMSA scan positive rate in patients with a longer total fever duration (39.5\%, $52.2 \%$, and 60.7 $\%$, respectively, $P=0.02$ ). When we analyzed the type of

Table 2. Clinical Comparison between DMSA Positive Group and Negative Group

\begin{tabular}{|c|c|c|c|}
\hline Groups & $\begin{array}{c}\text { DMSA positive } \\
\quad(n=141)\end{array}$ & $\begin{array}{l}\text { DMSA negative } \\
\quad(n=179)\end{array}$ & $P$ value \\
\hline Age (months) & $31 \pm 47$ & $17 \pm 35$ & 0.010 \\
\hline Male:Female & $74: 67$ & $110: 69$ & 0.102 \\
\hline \multicolumn{4}{|l|}{ Fever duration (days) } \\
\hline Prior to admission & $2.9 \pm 1.9$ & $2.7 \pm 1.9$ & 0.351 \\
\hline Total & $4.5 \pm 2.6$ & $3.5 \pm 2.1$ & $<0.001$ \\
\hline DMSA date (days)* & $2.7 \pm 1.1$ & $2.9 \pm 1.3$ & 0.133 \\
\hline \multicolumn{4}{|l|}{ Laboratory finding } \\
\hline \multicolumn{4}{|l|}{ Urine } \\
\hline Leukocyte esterase & $134(95)$ & $166(92.7)$ & 0.511 \\
\hline Nitrite & $60(42.6)$ & $63(35.2)$ & 0.182 \\
\hline Bacteriuria & $120(85.1)$ & $153(85.5)$ & 0.942 \\
\hline \multicolumn{4}{|l|}{ Blood } \\
\hline WBC $\left(/ \mathrm{mm}^{3}, \times 10^{3}\right)$ & $18.9 \pm 7.0$ & $14.8 \pm 5.5$ & $<0.001$ \\
\hline Neutrophil (\%) & $61 \pm 15$ & $50 \pm 17$ & $<0.001$ \\
\hline Lymphocyte (\%) & $28 \pm 14$ & $38 \pm 15$ & $<0.001$ \\
\hline Monocyte (\%) & $10 \pm 4$ & $10 \pm 6$ & 0.418 \\
\hline Hemoglobin (g/dL) & $11.3 \pm 1.3$ & $11.1 \pm 1.1$ & 0.202 \\
\hline Platelet $\left(/ \mathrm{mm}^{3}, \times 10^{3}\right)$ & $337 \pm 101$ & $369 \pm 109.8$ & 0.009 \\
\hline ESR $(\mathrm{mm} / \mathrm{h})$ & $58 \pm 32$ & $51 \pm 31$ & 0.062 \\
\hline CRP (mg/dL) & $8.0 \pm 5.4$ & $5.8 \pm 4.5$ & 0.001 \\
\hline Total protein (g/dL) & $6.6 \pm 0.6$ & $6.3 \pm 0.6$ & 0.002 \\
\hline Albumin (g/dL) & $4.2 \pm 0.4$ & $4.2 \pm 0.4$ & 0.945 \\
\hline
\end{tabular}

*DMSA date, duration from admission to the date of DMSA scan. defects seen in the DMSA scan according to fever duration, patients with a fever duration $\geq 8$ days had only a single defect, and no multiple defects or discrepant defects (Table 3).

\section{VCUG positive rate according to the type of defects on DMSA scan}

The VCUG positive rates in patients with a single defect, those with multiple defects, and those with discrepant defects were $22.4 \%$ (11/49), 60\% (9/15) and 70.6\% (12/17), respectively (Fig. 1). Patients with discrepant defects or multiple defects showed a significantly higher VCUG positive rates compared to those with a single defect $(P=0.04$ and $P=0.04$, respectively). There was no difference between the multiple defect and discrepant defect groups. With respect to the severity of VUR, there were 7 patients with mild VUR (grades I, II, and III) and 4 patients with severe VUR (grades IV and V) among the patients with a single defect, and there were 6 patients with mild VUR and 3 patients

Table 3. DMSA Positive Rates according to Total Fever Duration

\begin{tabular}{lcccc}
\hline Groups & $\begin{array}{c}<4 \text { days } \\
(n=223)\end{array}$ & $\begin{array}{c}5-7 \text { days } \\
(n=69)\end{array}$ & $\begin{array}{c}>8 \text { days } \\
(n=28)\end{array}$ & P value \\
\hline DMSA positive, $n(\%)$ & $88(39.5)$ & $36(52.2)$ & $17(60.7)$ & 0.02 \\
Single defect & $59(26.5)$ & $24(34.8)$ & $17(60.7)$ & \\
Multiple defects & $12(5.4)$ & $8(11.6)$ & $0(0)$ & \\
Discrepant defects & $17(7.5)$ & $4(5.8)$ & $0(0)$ & \\
\hline
\end{tabular}

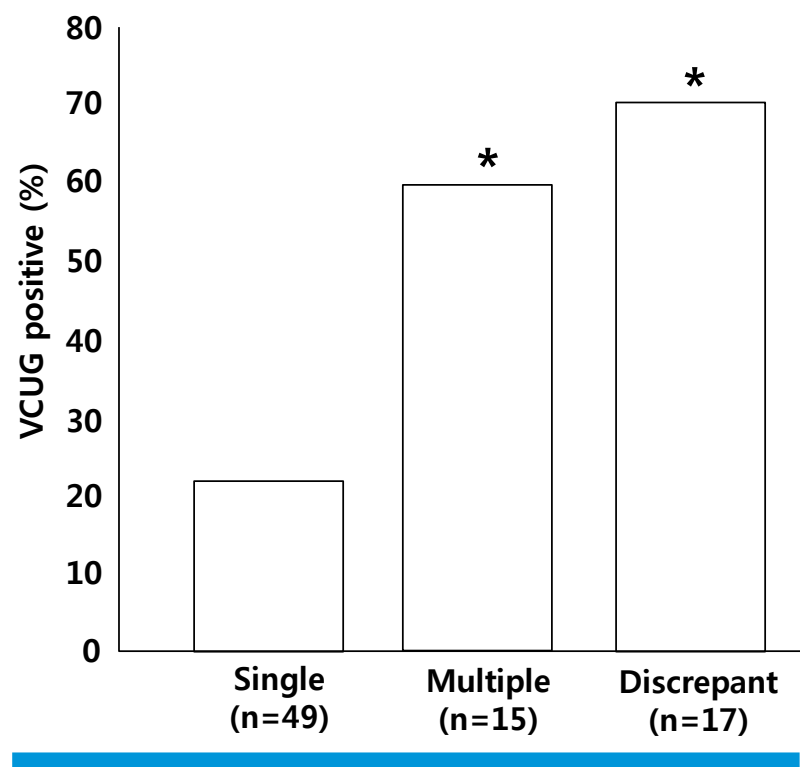

Figure 1. VCUG positive rates according to DMSA scan findings. ${ }^{*} P=0.04$, respectively, compared to single defect. 
www.chikd.org

with severe VUR in the patients with multiple defects. But, there were 4 patients with mild VUR and 8 patients with severe VUR among the patients with discrepant defects.

\section{Discussion}

In this study, we reconfirmed the fact that the majority of APN patients (79.4\%) were infants (0-1 year of age) with a male predominance (2.1:1), and children aged $>2$ years showed a marked female dominance (1:5). These demographic characteristics of APN are so unique that it is difficult to find similar phenomena in other infectious diseases or infection-related immune mediated diseases. It may be in part explained by the fact that an immature immune status during infancy may be associated with the pathogenesis of the disease. Interestingly, in contrast to other external pathogen infection such as tuberculosis and malaria ${ }^{10,11)}$, infants with APN in this series may have milder phenotypes compared to children, since the infant group had a shorter total fever duration, lower CRP value and DMSA scan positivity compared to the children group. It is possible that febrile infants with APN visited the hospital earlier and received early antibiotic treatment or the bacterial uropathogens in APN, including E.coli, are not external pathogens but they originate from the host ${ }^{3)}$.

DMSA is the agent of choice for renal scan (renal cortical scintigraphy). It is suggested that DMSA may bind to proximal tubule cell receptors through receptor-mediated endocytosis ${ }^{12}$. Since renal tubule cell receptors may have affinity for various substances from the glomerular ultrafiltrates or the local circulation, it is possible that some substances produced from the pathologic lesion(s) compete to binding sites. Also, it is possible that anatomical abnormalities affecting urinary flow or local circulation in the parenchyma of the kidneys may interfere with DMSA binding to renal tubular cells.

In the present study, the positive rate of a DMSA scan was $44.1 \%$. Other studies have reported various DMSA scan positive rates ranging from $32 \%$ to $79 \%{ }^{12-21)}$. However, the discrepant results in these studies may be influenced by the following factors: heterogeneity in the age of the subjects, indications for a DMSA scan, time between diagnosis of APN and performance of DMSA scan, diagnostic criteria
Huh SM, et al. • DMSA Scan in Childhood Acute Pyelonephritis 111

for DMSA scan, and patient selection biases. These findings indicate that DMSA scan has a limitation in detecting the pathologic lesions in the renal parenchyma, especially in infants, although it is certain that older children with APN and patients with VUR showed higher DMSA positivity.

Since it is believed that all patients with APN may have a focus where uropathogens replicate and produce inflammatory mediators, it is possible that patients with a negative DMSA scan may have small lesions in the early stage of APN, or it is more likely that they may have a lesion which produces lesser amounts of substances affecting DMSA binding to renal tubular cells. In this series, patients with positive DMSA scan had a longer total fever duration (4.5 days vs. 3.5 days) and higher values of inflammatory indices, including WBC, CRP and ESR, compared to patients with negative DMSA scan. Other studies have also reported that patients with positive DMSA scans had higher maximum temperature ${ }^{17,22)}$, prolonged fever duration (therapeutic delay time) ${ }^{14,16,18)}$, or severe laboratory values such as CRP and procalcitonin ${ }^{14,18,19,20.22)}$. Furthermore, we found that DMSA scan positivity was not associated with fever duration prior to admission (2.9 days vs. 2.7 days), but patients with a longer total fever duration had a higher DMSA scan positivity. Interestingly, all patients with a fever duration of $\geq 8$ days had a single defect on their DMSA scans, and none of the patients had multifocal defects or discrepant defects in this series. Our results suggest that all patients may have a lesion in the renal parenchyma in the beginning of the disease onset and patients with lesions which produce more inflammatory mediators that affect the DMSA scan experience more severe systemic inflammation, and that multiple defects or discrepant defects on a DMSA scan may be associated with anatomical abnormalities in the renal parenchyma.

VUR is an important factor that causes recurrent UTI and occasionally subsequent chronic kidney disease. However, VCUG for detecting VUR is not recommended for all patients with the first episode of APN because of several disadvantages such as the invasive method of study, radiation exposure, and low sensitivity. In general, VCUG is recommended in cases with risk factors such as positive renal US findings or DMSA scans ${ }^{23,24}$. We performed VCUG in selected patients who had severe abnormal findings on the US and/or DMSA scan. The positive rate of VUR in this 
study was $39.5 \%$ (32 cases/81 cases examined). The reported rates of VUR in patients with the first febrile UTI also ranged from $13 \%$ to $62 \%$ across studies ${ }^{13-18,20,21)}$. In these reports, the study results obtained from selected patients and those from all (unselected) patients with APN showed similar variations, suggesting that VCUG should only be performed in patients who are selected by severe abnormal findings on US and/or DMSA scan. In the present study, we classified DMSA lesions into 3 different types of cortical defects: single defect, multiple defects, and discrepant defects, and we found that the pattern of cortical defects seen on DMSA scan may indicate the presence of VUR. Patients with a single defect showed lower VUR positive rate (22.4\%) and patients with multiple defects or discrepant defects showed higher VUR positive rates (60\% and 70.6\%, respectively). Furthermore, patients with a high grade (grades IV and V) VURs were more frequently observed in the discrepant groups compared to that in patients with a single defect. Other studies regarding the prediction of VUR based on DMSA scan findings have also reported that patients with a positive DMSA scan had higher VUR rates compared to those without DMSA defects ${ }^{13,15,18,21)}$, and inversely, it could be interpreted that patients with a more severe VUR had a higher positivity and more severe DMSA scan findings. These findings also suggest that multiple defects or discrepant defects on a DMSA scan may be associated with anatomical abnormalities of the kidney rather than inflammatory pathologic lesions.

This study has some limitations. This study was conducted at a single center, and it was carried out retrospectively. This study did not include all febrile UTI patients admitted during the study period because of our definition of APN. Thus, patients who had pyuria and a positive urine culture but a CRP value $<2 \mathrm{mg} / \mathrm{dL}$ were excluded. The excluded patients were mostly infants who had a relative short fever duration prior to admission (1-2 days).

In conclusion, infants with APN may have milder phenotypes compared to children due to possibly early treatment. The positive rate of a DMSA scan was $44.1 \%$ of the subjects, and patients with positive DMSA were older, and had a longer total fever duration and a higher CRP value. However, there was no association between DMSA positivity and fever duration prior to admission. Multifocal or discrepant cortical defects on DMSA scan are related to a higher rate of VUR. These findings suggest that certain inflammatory mediators produced by a lesion or anatomical abnormalities in the renal parenchyma may be reflected in DMSA scan findings. Although DMSA scan has limitations in early diagnosis of APN, DMSA scan findings may aid in the prediction of the severity of systemic inflammation and detection of VUR.

\section{Conflicts of interest}

No potential conflict of interest relevant to this article was reported.

\section{References}

1. Hoberman A, Chao HP, Keller DM, Hickey R, Davis HW, Ellis D. Prevalence of urinary tract infection in febrile infants. J Pediatr 1993;123:17-23.

2. Elder JS. Urinary tract infection. In: In Behrman RE, Kliegman RM, Jenson HB (eds.), editors. Nelson textbook of Pediatrics. 20th ed. WB Saunders company, 2015:2556-67.

3. Lee KY. New insights for febrile urinary tract infection (acute pyelonephritis) in children. Child Kidney Dis 2016;20:37-44.

4. Edefonti A, Tel F, Testa S, De Palma D. Febrile urinary tract infections: clinical and laboratory diagnosis, imaging, and prognosis. Semin Nucl Med 2014;44:123-8

5. Berg UB, Johansson SB. Age as a main determinant of renal functional damage in urinary tract infection. Arch Dis Child 1983;58: 963-9.

6. Park YS. Renal scar formation after urinary tract infection in children. Korean J Pediatr 2012;55: 367-70.

7. Gordon I, Barkovics M, Pindoria S, Cole TJ, Woolf AS. Primary vesicoureteric reflux as a predictor of renal damage in children hospitalized with urinary tract infection: a systematic review and meta-analysis. J Am Soc Nephrol 2003;14:739-44.

8. Roberts KB. Revised AAP guideline on UTI in febrile infants and young children. Am Fam Physician 2012;86: 940-6.

9. Verrier-Jones K, Banerjee J, Boddy S-A, Grier D, Jadresic J, Tullus K, et al. Urinary tract infection in children: diagnosis, treatment and long-term management. August. 2007;NICE clinical guideline 54. www.nice.org.uk.

10. Jenkins HE, Yuen CM, Rodriguez CA, Nathavitharana RR, McLaughlin MM, Donald P, et al. Mortality in children diagnosed with tuberculosis: a systematic review and meta-analysis. Lancet Infect Dis 2017;17:285-95.

11. Tette EM, Neizer ML, Nyarko MY, Sifah EK, Sagoe-Moses IA, Nartey 
www.chikd.org

ET. Observations from Mortality Trends at The Children's Hospital, Accra, 2003-2013. PLoS One 2016;11:e0167947.

12. Weyer K, Nielsen R, Petersen SV, Christensen El, Rehling M, Birn H. Renal uptake of 99mTc-dimercaptosuccinic acid is dependent on normal proximal tubule receptor-mediated endocytosis. J Nucl Med 2013;54:159-65.

13. Benador D, Benador N, Slosman DO, Nusslé D, Mermillod B, Girardin E. Cortical scintigraphy in the evaluation of renal parenchyma changes in children with pyelonephritis. J Pediatr 1994;124:17-20.

14. Fernandez-Menendez JM, Malaga S, Matesanz JL, Solis G, Alonso S, Perez-Mendez C. Risk factors in the development of early technetium-99m dimercaptosuccinic acid renal scintigraphy lesions during first urinary tract infection in children. Acta Paediatr 2003;92:21-6.

15. Wu CY, Chiu PC, Hsieh KS, Chiu CL, Shih CH, Chiou YH. Childhood urinary tract infection: a clinical analysis of 597 cases. Acta Paediatr Taiwan 2004:45:328-33.

16. Jung Jl, Lim DH, Yim HE, Park MS, Yoo KH, Hong YS, et al. Fever duration and renal scar in pediatric urinary tract infection. J Korean Soc Pediatr Nephrol 2008;12:70-7.

17. Ataei N, Madani A, Habibi R, Khorasani M. Evaluation of acute pyelonephritis with DMSA scans in children presenting after the
Huh SM, et al. • DMSA Scan in Childhood Acute Pyelonephritis 113

age of 5 years. Pediatr Nephrol 2005;20:1439-44.

18. Pecile P, Miorin E, Romanello C, Vidal E, Contardo M, Valent F, et al. Age-related renal parenchymal lesions in children with first febrile urinary tract infections. Pediatrics 2009;124:23-9.

19. Kotoula A, Gardikis S, Tsalkidis A, Mantadakis E, Zissimopoulos A, Kambouri K, et al. Procalcitonin for the early prediction of renal parenchymal involvement in children with UTI: preliminary results. Int Urol Nephrol 2009;41:393-9.

20. Oh MM, Kim JW, Park MG, Kim JJ, Yoo KH, Moon DJ. The impact of therapeutic delay time on acute scintigraphic lesion and ultimate scar formation in children with first febrile UTI. Eur J Pediatr 2012;171:565-70.

21. Zhang X, Xu H, Zhou L, Cao Q, Shen Q, Sun L, et al. Accuracy of early DMSA scan for VUR in young children with febrile UTI. Pediatrics 2014;133:e30-8.

22. An YK, Cho MH, Kim KS. Which factors related to the renal cortical defects in infants under 3 months of age with urinary tract infections? Child Kidney Dis 2016;20:57-62.

23. Tullus K. Pediatrics: AAP recommends reduced imaging after first febrile UTI. Nat Rev Urol 2012;9:11-2.

24. Lee SJ. Clinical guideline for childhood urinary tract infection (Second Revision). Child Kidney Dis 2015;19:56-64. 